\title{
Optimal control problem on COVID-19 disease transmission model considering medical mask, disinfectants and media campaign
}

\author{
Dipo Aldila* \\ Department of Mathematics, Indonesia University, Depok 16424, Indonesia
}

\begin{abstract}
In this paper, a system of ordinary differential equation approach is developed to understand the spread of COVID-19. We first formulate the dynamic model by dividing the human population based on their health status, awareness status, and also including the free virus on the environment. We provide a basic analysis of the model regarding the wellposed properties and how the basic reproduction number can be used to determine the final state of COVID-19 in the population. A Pontryagin Maximum's Principle used to construct the model as an optimal control problem in a purpose to determine the most effective strategies against the spread of COVID-19. Three control strategies involved in the model, such as media campaign to develop an awareness of individuals, medical masks to prevent direct transmission, and use of disinfectant to reduce the number of free virus in the environment. Through numerical simulations, we find that the time-dependent control succeeds in reducing the outbreak of COVID-19. Furthermore, if the intervention should be implemented as a single intervention, then the media campaign gives the most effective cost strategy.
\end{abstract}

\section{Introduction}

Covid-19 has been found initially in Wuhan China, at the end of 2019 [1,2]. The symptoms of COVID-19 are like ordinary flu, but it could lead to death caused by difficulties in breathing for patients in chronic stages. Until July 2020, there is no medicine to cure infected individuals, and also no vaccine to prevent infection of COVID-19. However, several candidates for vaccines to prevent COVID-19 infection have been proposed and under consideration in several countries [3-5]. Hence, the most popular way to control the spread of COVID-19 is to prevent infection by introducing a non-pharmaceutical intervention, such as physical distancing, use of medical mask, quarantine, improvement of medical treatment, use of disinfectant, contact tracing and many more [6-8].

Many authors have used mathematical models to understand how diseases may spread among the population [9-13]. For the COVID-19 model, the first mathematical model that has been published to understand how COVID-19 spread in Indonesia is given in [14]. They predict how COVID-19 behave in Indonesia by using some estimated parameters from other

* Corresponding author : aldiladipo@sci.ui.ac.id 
countries. The author in [7] proposed a $S E A_{u} A_{d} I R$ model to describe how COVID-19 spread in Jakarta, where mathematical analysis regarding stability analysis conducted, and parameter estimation provided to see the impact of social distancing in Jakarta. Authors in $[14,15]$ also use a deterministic model to predict the spread of COVID-19 in Indonesia, and several other countries.

In this paper, we develop a mathematical model of COVID-19 spread among population considering two possible sources of infection, namely direct infection due to contact between susceptible human and infected human, and indirect transmission due to susceptible human touch an object which has a COVID-19 virus on it surfaces. Mathematical analysis for some basic properties conducted and followed with an optimal control problem of the model. The main purposes of this article are to determine the best strategies which combine three intervention: media campaign, medical mask, and disinfectant, against COVID-19.

The paper is organized as follows. In section 2, we construct the optimal control model. Mathematical analysis conducted in section 3 regarding the COVID-19 free equilibrium and the basic reproduction number. The optimal control characterization conducted in Section 4, followed by numerical experiments in Section 5. The last section is devoted to our conclusions.

\section{The $S_{u} S_{a} I R S-V$ model}

We consider the $S_{u} S_{a} I R S-V$ model in this article, to model how COVID-19 spread among population under three different intervention strategies, namely media campaign to develop awareness of individual, medical mask to prevent human-to-human transmission, and disinfectant to reduce number of free virus in the environment. Let $S_{u}$ and $S_{a}$ describe unaware and aware susceptible population, respectively, while $I$ and $R$ describe infected and recovered population, respectively. Furthermore, we denote $V$ as the free virus in the environment. The transmission diagram of the model described in Fig.1 below.

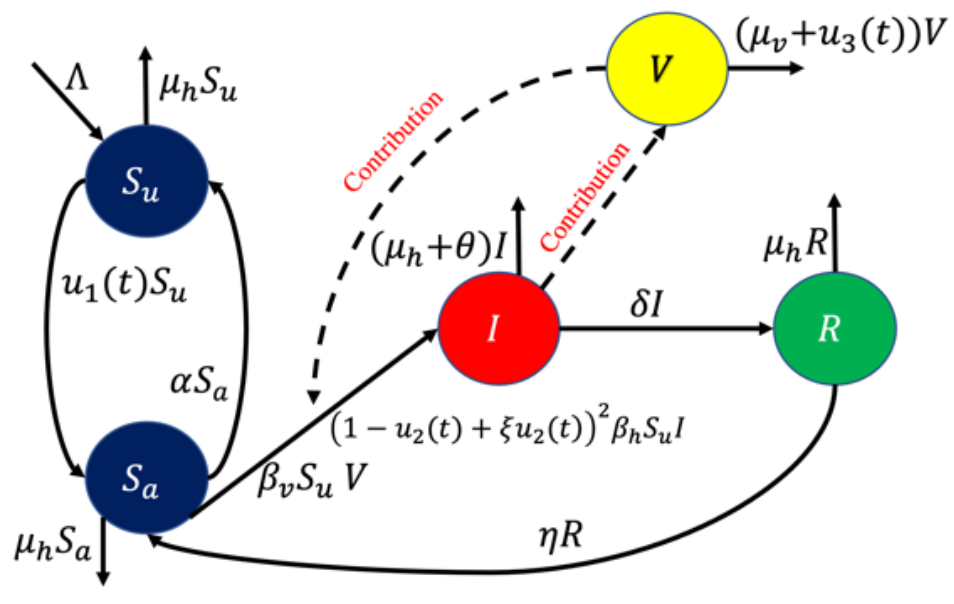

Figure 1. Transmission diagram of COVID-19 for system (1).

The following assumptions used to construct the model.

a. The population are closed. All migration is neglected.

b. Infected individual may death due to COVID-19.

c. Media campaign given in a rate of $u_{1}$ to increase human awareness. We assume that aware susceptible individual conduct a perfect self-isolation to avoid human-to-human infection. 
d. Due to assumption (c), only unaware susceptible individual who may get infected by COVID-19.

e. $u_{2}$ proportion of unaware susceptible and infected individual using medical mask to prevent human-to-human transmission, while the rest $\left(1-u_{2}\right)$ do not.

f. Disinfectant conducted with a rate of $u_{3}$ to reduce number of free virus in the environment.

Based on previous assumptions and transmission diagram in Figure 1, the COVID-19 transmission model is expressed by the following system of ordinary differential equations:

$$
\begin{aligned}
& \frac{d S_{u}}{d t}=\Lambda-\left(1-u_{2}(t)+\xi u_{2}(t)\right)^{2} \beta_{h} S_{u} I-\beta_{v} S_{u} V-\mu_{h} S_{u}-u_{1}(t) S_{u}+\alpha S_{a}, \\
& \frac{d S_{a}}{d t}=u_{1}(t) S_{u}-\alpha S_{a}-\mu_{h} S_{a}+\eta R, \\
& \frac{d I}{d t}=\left(1-u_{2}(t)+\xi u_{2}(t)\right)^{2} \beta_{h} S_{u} I+\beta_{v} S_{u} V-\delta I-\mu_{h} I-\theta I, \\
& \frac{d R}{d t}=\delta I-\mu_{h} R-\eta R, \\
& \frac{d V}{d t}=\omega I-\mu_{v} V-u_{3}(t) V,
\end{aligned}
$$

which supplemented with a non-negative initial condition.

Our aim is to minimized number of infected individuals, with as low as possible cost for intervention. The objective functional to be minimized is given by

$$
J\left(u_{1}, u_{2}, u_{3}, I\right)=\int_{0}^{T}\left(c I+d_{1} u_{1}^{2}+d_{2} u_{2}^{2}+d_{3} u_{3}^{2}\right) d t,
$$

where $c, d_{i}$ for $i=1,2,3$ is the weight parameter for infected individuals and control parameters, respectively, and $T$ is the final time. Description and range of value for each parameter in system (1) is given in Table 1.

Table 1. Description of parameters for COVID-19 model (1).

\begin{tabular}{|c|l|c|c|}
\hline Par. & \multicolumn{1}{|c|}{ Description } & Value & Ref. \\
\hline$\Lambda$ & Natural recruitment rate due to new born & $\frac{49316712}{70 \times 365}$ & {$[29]$} \\
\hline$u_{1}$ & Media campaign rate & {$[0,1]$} & Assumed \\
\hline$u_{2}$ & Proportion of medical mask & {$[0,1]$} & Assumed \\
\hline$u_{3}$ & Rate of disinfectant & $1.26 \times 10^{-8}$ & Assumed \\
\hline$\beta_{h}$ & Human-to-human transmission rate & $8 \times 10^{-11}$ & {$[8]$} \\
\hline$\beta_{v}$ & $\begin{array}{l}\text { Transmission rate from free virus in the } \\
\text { environment }\end{array}$ & $\frac{1}{70 \times 365}$ & {$[30]$} \\
\hline$\mu_{h}$ & Natural death rate of human & $\frac{1}{2}$ & {$[8]$} \\
\hline$\mu_{v}$ & Survival rate of virus without host & 0.1 & {$[29]$} \\
\hline$\alpha$ & Drop-out rate from aware population & 0.12978 & {$[31]$} \\
\hline$\delta$ & Natural recovery rate & 0.015 & {$[31]$} \\
\hline$\theta$ & Death rate due to Covid-19 & 10000 & {$[8]$} \\
\hline$\omega$ & $\begin{array}{l}\text { Average production rate of virus from infected } \\
\text { individuals }\end{array}$ & $\frac{1}{30}$ & Assumed \\
\hline$\eta$ & Transition rate due to temporal immunity vanished & {$[0,1]$} & Assumed \\
\hline$\xi$ & Medical mask efficacy & & \\
\hline
\end{tabular}




\section{Analysis on COVID-19 free equilibrium and $\mathcal{R}_{\mathbf{0}}$}

To analyse the existence, and stability of the equilibrium points, let us assume that all intervention strategy are constant, hence $u_{j}(t)=u_{i}$ for $j=1,2,3$.

For COVID-19 model (1) to be epidemiologically meaningful, it is important to prove that all it states variables are non-negative for all time $t>0$, as long as the initial conditions are non-negative. This properties given in the following Lemma 1.

Lemma 1. Let the initial condition $S_{u}(0) \geq 0, S_{a}(0) \geq 0, I(0) \geq 0, R(0) \geq 0$, and $V(0) \geq$ 0 . Then the solutions for all variables of COVID-19 model in (1) are non-negative for all time $t>0$.

Invariant Regions. It can be seen that our COVID-19 model will be analyzed in the following region. Consider the feasible region:

$$
\Omega=\Omega_{\mathrm{h}} \times \Omega_{v} \subset \mathbb{R}_{+}^{4} \times \mathbb{R}_{+},
$$

where

$$
\Omega_{h}=\left\{\left(S_{u}(t), S_{a}(t), I(t), R(t)\right) \in \mathbb{R}_{+}^{4}: N_{h}(t) \leq \frac{\Lambda}{\mu_{h}}\right\},
$$

with $S_{u}(t)+S_{a}(t)+I(t)+R(t)=N_{h}(t)$ and

$$
\Omega_{v}=\left\{V \in \mathbb{R}_{+}: V \leq \frac{\omega \Lambda}{\mu_{h}\left(\mu_{v}+u_{3}\right)}\right\}
$$

Lemma 2. The region $\Omega \subset \mathbb{R}_{+}^{5}$ is positively-invariant for the model (1) with non-negative initial conditions in $\mathbb{R}_{+}^{5}$.

Next, we will determine the form of equilibrium points. The COVID-19 free-equilibrium point of system(1) is given by

$$
E_{0}=\left(S_{u}, S_{a}, I, R, V\right)=\left(\frac{\Lambda\left(\alpha+\mu_{h}\right)}{\mu_{h}\left(\alpha+\mu_{h}+u_{1}\right)}, \frac{\Lambda u_{1}}{\mu_{h}\left(\alpha+\mu_{h}+u_{1}\right)}, 0,0,0\right) .
$$

Using the next-generation matrix approach [17], the basic reproduction number of system (1), denoted by $\mathcal{R}_{0}$, is given by

$$
\mathcal{R}_{0}=\mathcal{R}_{h}+\mathcal{R}_{v}
$$

where $\quad \mathcal{R}_{h}=\frac{\Lambda\left(\alpha+\mu_{h}\right)\left(1-u_{2}(t)+\xi u_{2}(t)\right)^{2} \beta_{h}}{\mu_{h}\left(\alpha+\mu_{h}+u_{1}\right)\left(\delta+\mu_{h}+\theta\right)}, \quad$ and $\quad \mathcal{R}_{v}=\frac{\Lambda\left(\alpha+\mu_{h}\right) \omega \beta_{v}}{\mu_{h}\left(\alpha+\mu_{h}+u_{1}\right)\left(\delta+\mu_{h}+\theta\right)\left(\mu_{v}+u_{3}\right)}$. Basic reproduction number represent number of new infected individual caused by a primary infected individual during it infection period in a completely susceptible individuals. Note that $\mathcal{R}_{h}$ represent the basic reproduction number due to human-to-human infection, while $\mathcal{R}_{v}$ is the basic reproduction number due to contact with free virus in the environment.

Using Theorem 2 in [18], the following results established.

Lemma 3. The Covid-19 free equilibrium point $E_{0}$ of system (1) is locally asymptotically stable $(L A S)$ if $\mathcal{R}_{0}<1$ and unstable if $\mathcal{R}_{0}>1$.

Lemma 3 is a common result in an epidemiology model for disease spread. Please see $[25,26,27]$ for a similar result regarding the LAS properties of the disease-free equilibrium point for other disease models. 


\section{Effect of controls on the basic reproduction number.}

From Lemma 3, it can be seen that COVID-19 will disappeared from the population whenever $\mathcal{R}_{0}<1$. Hence, reducing $\mathcal{R}_{0}$ will increase the chance to eradicate COVID-19 from the environment. Since

$$
\frac{\partial \mathcal{R}_{0}}{\partial u_{1}}=-\frac{\Lambda\left(\alpha+\mu_{h}\right)\left(1-u_{2}(t)+\xi u_{2}(t)\right)^{2} \beta_{h}}{\mu_{h}\left(\alpha+\mu_{h}+u_{1}\right)^{2}\left(\delta+\mu_{h}+\theta\right)}-\frac{\Lambda\left(\alpha+\mu_{h}\right) \omega \beta_{v}}{\mu_{h}\left(\alpha+\mu_{h}+u_{1}\right)^{2}\left(\delta+\mu_{h}+\theta\right)\left(\mu_{v}+u_{3}\right)}<0,
$$

we conclude that increasing $u_{1}$ will reduce $\mathcal{R}_{0}$. Similarly, since

$$
\frac{\partial \mathcal{R}_{0}}{\partial u_{2}}=-\frac{\Lambda\left(\alpha+\mu_{h}\right) 2\left(1-u_{2}(t)+\xi u_{2}(t)\right)(1-\xi) \beta_{h}}{\mu_{h}\left(\alpha+\mu_{h}+u_{1}\right)\left(\delta+\mu_{h}+\theta\right)}<0,
$$

we conclude that increasing $u_{2}$ will reduce $\mathcal{R}_{0}$. However, it can be seen that unlike $u_{1}$ where the implementation could reduce $\mathcal{R}_{h}$ and $\mathcal{R}_{v}$ simultaneously, implementation of $u_{2}$ only reducing $\mathcal{R}_{h}$. The reason simply because $u_{2}$ conducted only to reduce human-to-human infection rate. The other results is regarding the disinfectant intervention. Since

$$
\frac{\partial \mathcal{R}_{0}}{\partial u_{3}}=-\frac{\Lambda\left(\alpha+\mu_{h}\right) \omega \beta_{v}}{\mu_{h}\left(\alpha+\mu_{h}+u_{1}\right)\left(\delta+\mu_{h}+\theta\right)\left(\mu_{v}+u_{3}\right)^{2}}<0,
$$

we also can conclude that disinfectant could reduce $\mathcal{R}_{0}$. Since the purposes of disinfectant $u_{3}$ is to reduce number of free virus on the environment, we have that $u_{3}$ only affect $\mathcal{R}_{v}$.

We close this section with the following remark.

Remark 1. The basic reproduction number of the COVID-19 model in (1) generated by two source of infection, namely human-to-human and environmental transmission. All intervention (media campaign, medical mask and disinfectant) are promising to control the spread of COVID-19 since they could reduce $\mathcal{R}_{0}$. However, only intervention of media campaign that could reduce both component of $\mathcal{R}_{0}$, while medical mask only reducing human-to-human transmission, and disinfectant only reducing environmental transmission.

\section{Optimal control characterization}

Our goal is to minimize the cost function in (2) subject to system (1). As mentioned before, the cost function in (2) represent the problem of minimizing number of infected individual $\left(\int_{0}^{T}(c I) d t\right)$, and cost for interventions $\left(\int_{0}^{T}\left(d_{1} u_{1}^{2}+d_{2} u_{2}^{2}+d_{3} u_{3}^{2}\right) d t\right)$. In this paper, a quadratic objective functional is implemented to measuring the control cost, since we assume that the cost for intervention are nonlinear. This quadratic cost function has been frequently used by many authors $[8,10,19,20,21]$.

We seek to find optimal controls $u_{1}^{*}, u_{2}^{*}$, and $u_{3}^{*}$ such that

$$
J\left(u_{1}^{*}, u_{2}^{*}, u_{3}^{*}\right)=\min _{U}\left\{J\left(u_{1}, u_{2}, u_{3}\right)\right\},
$$

where the control set $U$ is given by

$$
U=\left\{\left(u_{1}, u_{2}, u_{3}\right):[0, T] \rightarrow[0,1]\right\} .
$$

The necessary condition for control parameters must satisfy the Pontryagin's Maximum principle [22]. First, let us formulate the Hamiltonian from the cost function (2) and the COVID-19 dynamic model (1) to obtain the optimality conditions. Let us define the Hamiltonian for system (1) as follows 


$$
\begin{aligned}
& H=c I+d_{1} u_{1}^{2}+d_{2} u_{2}^{2}+d_{3} u_{3}^{2} \\
& +\lambda_{1}\left(\Lambda-\left(1-u_{2}(t)+\xi u_{2}(t)\right)^{2} \beta_{h} S_{u} I-\beta_{v} S_{u} V-\mu_{h} S_{u}-u_{1}(t) S_{u}+\alpha S_{a}\right) \\
& +\lambda_{2}\left(u_{1}(t) S_{u}-\alpha S_{a}-\mu_{h} S_{a}+\eta R\right) \\
& +\lambda_{3}\left(\left(1-u_{2}(t)+\xi u_{2}(t)\right)^{2} \beta_{h} S_{u} I+\beta_{v} S_{u} V-\delta I-\mu_{h} I-\theta I\right) \\
& +\lambda_{4}\left(\delta I-\mu_{h} R-\eta R\right) \\
& +\lambda_{5}\left(\omega I-\mu_{v} V-u_{3}(t) V\right),
\end{aligned}
$$

where $\lambda_{i}$ for $i=1,2,3,4,5$ are the associated adjoints for state $S_{u}, S_{a}, I, R, V$, respectively.

Theorem 1. Given an optimal controls $u_{1}^{*}, u_{2}^{*}, u_{3}^{*}$ and solutions $S_{u}^{*}, S_{a}^{*}, I^{*}, R^{*}, V^{*}$ of the corresponding state system (1) that minimizes $J\left(u_{1}^{*}, u_{2}^{*}, u_{3}^{*}, I^{*}\right)$ over $U$. Then there exists adjoint variables $\lambda_{i}$ for $i=1,2,3,4,5$ which satisfying

$$
-\frac{d \lambda_{1}}{d t}=\frac{\partial H}{\partial S_{u}},-\frac{d \lambda_{2}}{d t}=\frac{\partial H}{\partial S_{a}},-\frac{d \lambda_{3}}{d t}=\frac{\partial H}{\partial I},-\frac{d \lambda_{4}}{d t}=\frac{\partial H}{\partial R},-\frac{d \lambda_{5}}{d t}=\frac{\partial H}{\partial V}
$$

And with transversality conditions $\lambda_{i}(T)=0$ for $i=1,2,3,4,5$. The optimality conditions are given as

$$
\frac{\partial H}{\partial u_{j}}=0, j=1,2,3
$$

Taking the lower bound and upper bound for each control variables as 0 and 1, respectively, the control $u_{1}^{*}, u_{2}^{*}$, and $u_{3}^{*}$ is given by

$$
\begin{gathered}
u_{1}^{*}=\min \left\{1, \max \left\{0, \frac{S_{u}\left(\lambda_{1}-\lambda_{2}\right)}{2 d_{1}}\right\}\right\}, \\
u_{2}^{*}=\min \left\{1, \max \left\{0, \frac{\beta_{h} S_{u} I(1-\xi)\left(\lambda_{1}-\lambda_{3}\right)}{\beta_{h} S_{u} I(1-\xi)^{2}\left(\lambda_{1}-\lambda_{3}\right)-d_{2}}\right\}\right\}, \\
u_{3}^{*}=\min \left\{1, \max \left\{0, \frac{V \lambda_{5}}{2 d_{3}}\right\}\right\} .
\end{gathered}
$$

Proof. The existence of an optimal control is guaranteed using the result by author in [23]. The adjoint system satisfying the following ordinary differential equations

$$
\begin{aligned}
& \frac{d \lambda_{1}}{d t}=\left(\beta_{h} I\left(1-u_{2}+\xi u_{2}\right)^{2}+\beta_{v} V\right)\left(\lambda_{1}-\lambda_{3}\right)+u_{1}\left(\lambda_{1}-\lambda_{2}\right)+\mu_{h} \lambda_{1} \\
& \frac{d \lambda_{2}}{d t}=\alpha\left(\lambda_{2}-\lambda_{1}\right)+\mu_{h} \lambda_{2} \\
& \frac{d \lambda_{3}}{d t}=-C+\beta_{h} S_{u}\left(1-u_{2}+\xi u_{2}\right)^{2}\left(\lambda_{1}-\lambda_{3}\right)+\delta\left(\lambda_{3}-\lambda_{4}\right)+\left(\theta+\mu_{h}\right) \lambda_{3}-\omega \lambda_{5} \\
& \frac{d \lambda_{4}}{d t}=\eta\left(\lambda_{4}-\lambda_{2}\right)+\mu_{h} \lambda_{4} \\
& \frac{d \lambda_{5}}{d t}=\beta_{v} S_{u}\left(\lambda_{1}-\lambda_{3}\right)+\left(\mu_{v}+u_{3}\right) \lambda_{5}
\end{aligned}
$$

With a transversality conditions $\lambda_{i}(T)=0$ for $i=1,2,3,4,5$. Optimality condition (5) is taken from $\frac{\partial H}{\partial u_{j}}=0, j=1,2,3$, solve each of it respect to $u_{j}$, and combined it with it correspond lower and upper bound. 


\section{Numerical experiments}

To In order to solve the optimal control problem (1) with objective functional (2), we used an iterative forward-backward method. At first, we give an initial guess for control variables $u_{j}$ for $j=1,2,3$, and solve the state system (1) forward in time using the Runge-Kutta method with initial condition of each state variables given. Next, we calculate the adjoint system (6) backward in time with the transversality condition. We update the control variables using (5) and iterate this scheme until the convergence criteria achieved. Please see [8,20,21,24-28] for more example on the implementation of this scheme.

To conduct simulation on this section, we use parameter values as in Table 1 . When no control intervention given, the basic reproduction number of system (1) is 5.87 which indicates that the COVID-19 free equilibrium is unstable. The dynamic of susceptible unaware, susceptible aware, infected human and free virus is given in Figure (2). It can be seen that the infected individual is increasing and reach the outbreak at $t=18$ days, and start decreasing to it endemic equilibrium point for larger $t$.
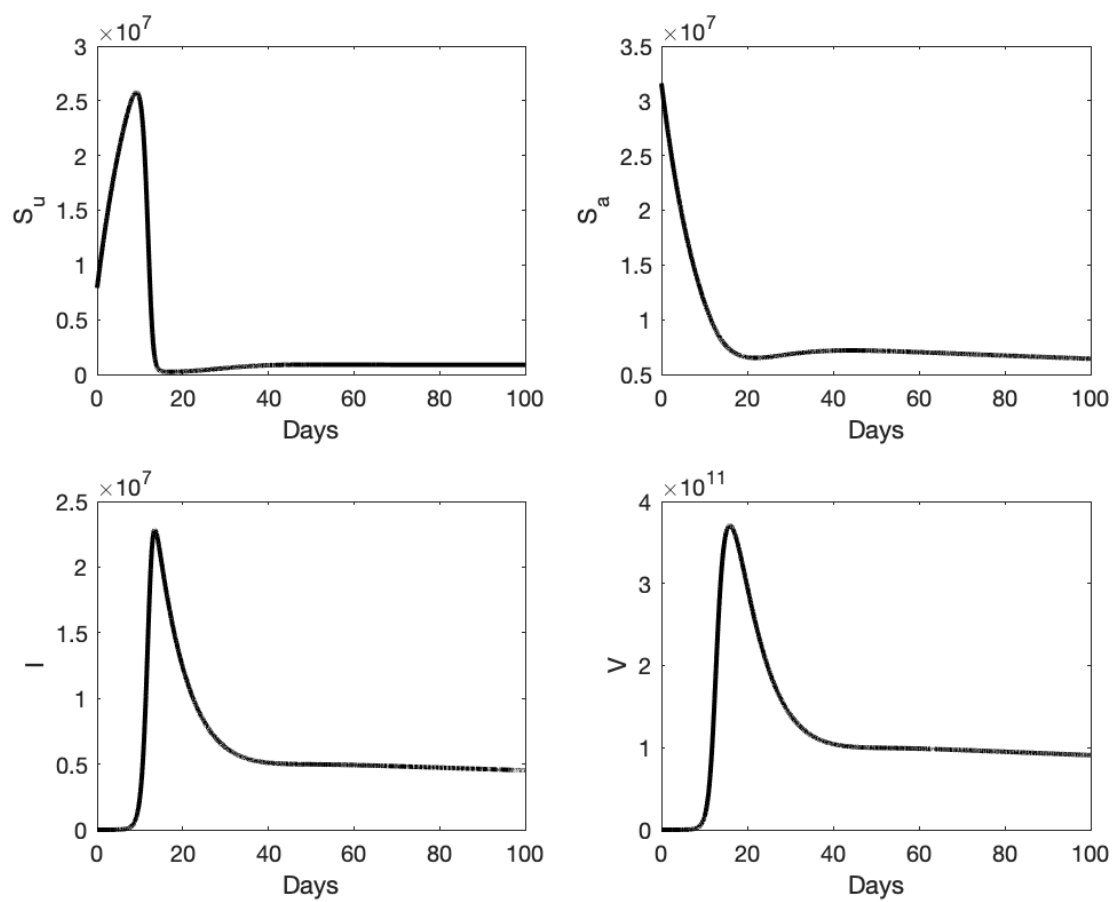

Figure 2. The dynamic of each variable in system (1) except $R$, where no control implemented.

The second simulation is conducted to simulate the effect intervention, when only media campaign implemented in the field, while medical mask and disinfectant ignored. Hence, we use $u_{1} \geq 0, u_{2}=u_{3}=0$. The result is given in Figure 3 . It can be seen that by implementing media campaign in it highest rate from the beginning of simulation time, number of infected individual cannot increase significantly. However, the effect of media campaign only succeed at $t \leq 30$ days, since after that, number of infected individual start to increase. Even though number of infected individual increases, but media campaign intervention succeeds to reduce the level of the outbreak, and delay it occurrence time. With this simulation, we have the cost function is $4.86 \times 10^{7}$, and number of infected individuals avoided are 2.594.699 
individuals. Hence, the average cost for each infected individual avoided is $\frac{4.86 \times 10^{7}}{2.594 .699}=$ 18.76.
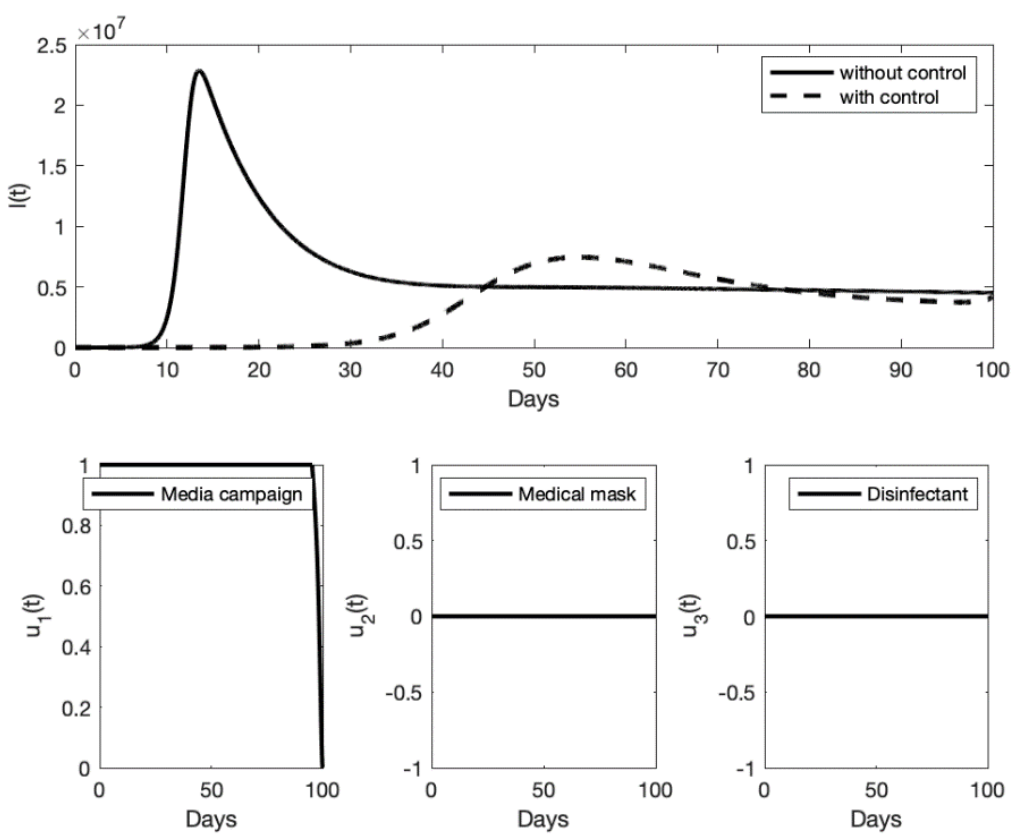

Figure 3. The second simulation. The dynamic of infected individual (above) when only media campaign intervention implemented, while other interventions ignored.

The third simulation is conducted to see the effect when only disinfectant as the only intervention. Hence, we set $u_{1}=0, u_{2}=0, u_{3} \geq 0$. The results can be seen in Figure 4 . It is easy to see that the effect of disinfectant in reducing number of infected individual is not as good as media campaign. The effect of disinfectant in Figure 4 is that it only reduce the outbreak slightly, and delay the occurrence of the outbreak for a moment. This is as the effect of control profile in Figure (4), With this simulation, number of infected avoided are 36.764 individual, whit cost function is $7.56 \times 10^{6}$. Hence, average cost for each infected individual avoided is 20.57 .

The last simulation (fourth simulation) is when all intervention implemented. In this simulation, all intervention implemented in the field. The dynamic of infected individuals along with all control variables is given in Figure 5. It can be seen that all interventions should be given it high rates / proportion from the beginning of simulation time, and start to decreasing when the outbreak have been passed. As a result of this scenario, the size of the outbreak has been reduced significantly, and it occurrence time have been delayed. The cost for this scenario is $1.72 \times 10^{7}$ with the total number of infected individual that have been avoided are 2.903.787 individuals. Hence, the average cost for each infected individual that have been avoided is 5.92 . 

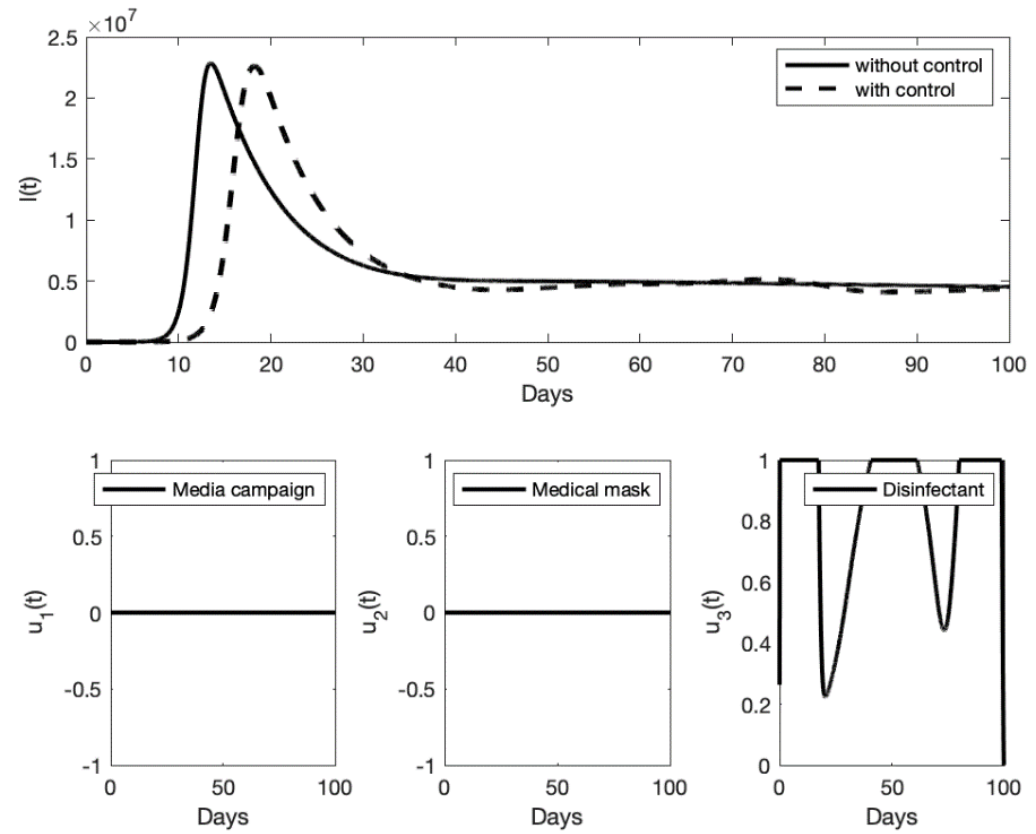

Figure 4. The third simulation. The dynamic of infected individual (above) when only disinfectant intervention implemented, while other interventions ignored.
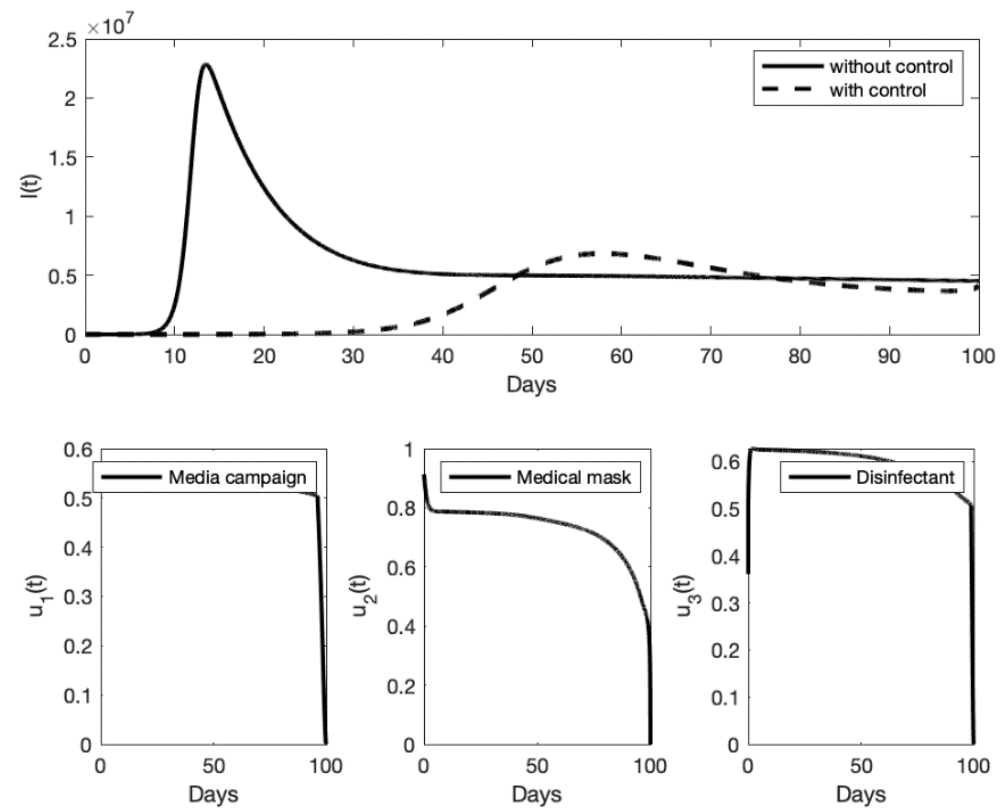

Figure 5. The fourth simulation. The dynamic of infected individual (above) when all interventions implemented. 


\section{Discussion and concluding remarks}

In this article, we introduce a simple deterministic model to understand the spread of COVID19 under some interventions: media campaign, medical masks, and a disinfectant. Each control has its specific purposes in controlling the spread of COVID-19. The model conducted a five-dimensional system of ordinary differential equations, where free coronavirus in the environment have been considered in the model.

Mathematical analysis regarding the COVID-19 free equilibrium and the basic reproduction number has been shown analytically. We find that COVID-19 will be eradicated from the field whenever the basic reproduction number is less than one. From analysis on the derivation of the basic reproduction number, we find that all derivative of the basic reproduction number to each control parameter is negative, which indicates the increased value of controls will reduce the size of the basic reproduction number. Hence, increasing the rate/proportion of controls will increase the chance to eradicate COVID-19 from the field.

From optimal control simulation, we show how all controls could reduce the number of infected individuals. Each simulation shows how interventions could reduce the size of the outbreak and delay the outbreak's occurrence. However, not all interventions succeed in reducing the spread of COVID-19 significantly. Using a single intervention, we find that the media campaign is more effective in reducing the number of infected individuals compared with using disinfectant only. From an analysis of the average cost for each individual that has been avoided for each scenario, we find that using all interventions is the most effective strategy since the average cost is only 5.92 , while using media campaign is 18.76 , and disinfectant only is 20.57 .

Although our results here indicate a promising intervention strategy to control COVID19 , we still have some limitations on this work. Our work not yet implemented in real incidence data. Hence, real practical in the field still need further justification. The second limitation is that many reports state that there are many undetected cases in the field. These undetected cases contribute to the infection process of COVID-19. Hence, for further work, accommodating undetected cases could be considered to modify our proposed model. For our previous work, which considers an undetected case, can be seen in $[7,29]$.

\section{Acknowledgements}

This research is financially supported by Indonesia University with PUTI Q1 research grant scheme 2020, ID Number: NKB-1380/UN2.RST/HKP.05.00/2020.

\section{References}

1. South China Morning Post Coronavirus: China's first confirmed Covid-19 case traced back to November 17. Published on Mar 13, 2020 and visited on June 29, (2020).

2. World Health Organization, Corona virus disease (COVID-19) Pandemic, https://www.who.int/emergencies/diseases/ novel-coronavirus-2019, Accessed on June 10, (2020).

3. The Jakarta Post : China's COVID-19 vaccine candidate shows promise in human trials, CNBG says, www.thejakartapost.com . Visited on August 3, (2020).

4. BBC : Coronavirus: Encouraging results in vaccine trials, www.bbc.com . Visited on August 3, (2020).

5. The Conversation : COVID-19 vaccine trial in South Africa: everything you need to know, https://theconversation.com/. Visited on August 3, (2020). 
6. Q. Bi, Y. Wu, S. Mei, C. Ye, X. Zou, Z. Zhang, X. Liu, L. Wei, S.A. Truelove, T. Zhang, et al., Epidemiology and transmission of COVID-19 in shenzhen China: Analysis of 391 cases and 1,286 of their close contacts, MedRxiv (2020).

7. D. Aldila, S. A. Khoshnaw, E. Safitri, et al., A mathematical study on the spread of COVID-19 considering social distancing and rapid assessment: The case of Jakarta, Indonesia, Chaos, Solitons and Fractals 139 (2020) 110042.

8. D. Aldila, Cost effectiveness analysis and backward bifurcation analysis on COVID-19 transmission model considering direct and indirect transmission, Commun. Math. Biol. Neurosci. 2020, (2020):X, https://doi.org/10.28919/cmbn/4779.

9. Wijaya, K.P., Páez Chávez, J., Aldila, D., An epidemic model highlighting humane social awareness and vector-host lifespan ratio variation, Communications in Nonlinear Science and Numerical Simulation 90,105389 (2020).

10. Aldila, D., Handari, B.D., Widyah, A., Hartanti, G., Strategies of optimal control for hiv spreads prevention with health campaign, Communications in Mathematical Biology and Neuroscience (2020), 7.

11. Aldila, D., Seno, H., A Population Dynamics Model of Mosquito-Borne Disease Transmission, Focusing on Mosquitoes' Biased Distribution and Mosquito Repellent Use, Bulletin of Mathematical Biology 81(12), pp. 4977-5008 (2019).

12. Putri, Y.E., Rozi, S., Tasman, H., Aldila, D., Assessing the effect of extrinsic incubation period (EIP) prolongation in controlling dengue transmission with wolbachia-infected mosquito intervention, AIP Conference Proceedings 1825,020019 (2017).

13. Maimunah, Aldila, D., Mathematical model for HIV spreads control program with ART treatment, Journal of Physics: Conference Series 974(1),012035 (2018).

14. Nuraini, N., Khairuddin, K., and Apri, M., Data and Simulation of COVID-19 in Indonesia based on Mathematical Modeling Approach, Communication in Biomathematical Sciences, Vol. 3, No. 1, (2020), pp 1-8.

15. Soewono, E., On the Analysis of Covid-19 Transmission in Wuhan, Diamond Princess and Jakarta-cluster, Communication in Biomathematical Sciences, Vol. 3, No. 1, (2020), pp 9-18.

16. Ndii, M., Z., Hadisoemarto, P., Agustian, D., \& Supriatna, A., K., “An analysis of Covid19 transmission in Indonesia and Saudi Arabia", Communication in Biomathematical Sciences, Vol. 3, No. 1, (2020), pp 19-27.

17. O. Diekmann, J. A. P. Heesterbeek, and M. G. Roberts, "The construction of nextgeneration matrices for compartmental epidemic models," Journal of the Royal Society Interface, vol. 7, no. 47, pp. 873-885, (2010).

18. P. V. Driessche, J. Watmough, Reproduction numbers and sub-threshold endemic equilibria for compartmental models of disease transmission, Mathematical Biosciences (2002) 29-48.

19. Agusto FB, ELmojtaba IM. Optimal control and cost-effective analysis of malaria/visceral leishmaniasis co-infection. PLoS One. (2017);12(2):e0171102. Published 2017 Feb 6. doi:10.1371/journal.pone.0171102.

20. Handari, B. D., Vitra, F., Ahya, R., \& Aldila, D., Optimal control in a malaria model: intervention of fumigation and bed nets, Advances in Difference Equations, 2019, 1, 497, (2019).

21. Aldila,D.,Padma,H.,Khotimah,K.,Handari,B.D.,Tasman,H.:Analyzing the MERS control strategy through an optimal control problem. Int. J. Appl. Math. Comput. Sci. 28(1), 169-184 (2018). 
22. L.S. Pontryagin, V.G. Boltyanskii, R.V. Gamkrelidze, E.F. Mishchenko, The Mathematical Theory of Optimal Processes, Wiley, New York, (1962).

23. W.H. Fleming and R.W. Rishel. Deterministic and stochastic optimal control, vol- ume 1. Springer Science \& Business Media, (2012).

24. Aldila, D., Handari, B.D., Widyah, A., Hartanti, G., Strategies of optimal control for hiv spreads prevention with health campaign, Communications in Mathematical Biology and Neuroscience 2020,7 (2020).

25. Aldila, D., Nareswari, K., Tasman, H., An optimum control model for resistance fumigation for dengue, AIP Conference Proceedings 2021,060001 (2018).

26. Hafidh, E.P., Aulida, N., Handari, B.D., Aldila, D., Optimal control problem from tuberculosis and multidrug resistant tuberculosis transmission model, AIP Conference Proceedings 2023,020223 (2018).

27. Rohman, M.I.S., Handari, B.D., Aldila, D., An impulse fumigation scenario to control dengue spreads, AIP Conference Proceedings 2023,020213 (2018).

28. Erwina, N., Aldila, D., Soewono, E., Optimal control of diarrhea transmission in a flood evacuation zone, AIP Conference Proceedings 1589, pp. 476-479 (2014).

29. D. Aldila, Analyzing the impact of the media campaign and rapid testing for COVID-19 as an optimal control problem in East Java, Indonesia, Under review in Chaos, Solitons and Fractals, (2020).

30. Feng Z, Velasco-Hernndez JX. Competitive exclusion in a vector-host model for the dengue fever. J Math Biol. (1997);35(5):523-544. doi:10.1007/s002850050064.

31. N.M. Ferguson, D. Laydon, G. Nedjati-Gilani, N. Imai, K. Ainslie, M. Baguelin, S. Bhatia, A. Boonyasiri, Z. Cucunuba, G. Cuomo-Dannenburg, et al., Impact of NonPharmaceutical Interven- tions (NPIs) to Reduce COVID-19 Mortality and Healthcare Demand, vol. 16, Imperial College COVID-19 Response Team, London, (2020). 